\title{
Traditional botanical knowledge of artisanal fishers in southern Brazil
}

\author{
Marcela Meneghetti Baptista ${ }^{1}$, Marcelo Alves Ramos², Ulysses Paulino de Albuquerque ${ }^{3}$, \\ Gabriela Coelho-de-Souza ${ }^{4}$ and Mara Rejane Ritter ${ }^{5^{*}}$
}

\begin{abstract}
Background: This study characterized the botanical knowledge of artisanal fishers of the Lami community, Porto Alegre, southern Brazil based on answers to the following question: Is the local botanical knowledge of the artisanal fishers of the rural-urban district of Lami still active, even since the district's insertion into the metropolitan region of Porto Alegre?
\end{abstract}

Methods: This region, which contains a mosaic of urban and rural areas, hosts the Lami Biological Reserve (LBR) and a community of 13 artisanal fisher families. Semi-structured interviews were conducted with 15 fishers, complemented by participatory observation techniques and free-lists; in these interviews, the species of plants used by the community and their indicated uses were identified.

Results: A total of 111 species belonging to 50 families were identified. No significant differences between the diversities of native and exotic species were found. Seven use categories were reported: medicinal (49\%), human food (23.2\%), fishing (12.3\%), condiments (8\%), firewood (5\%), mystical purposes (1.45\%), and animal food (0.72\%). The medicinal species with the highest level of agreement regarding their main uses (AMUs) were Aloe arborescens Mill., Plectranthus barbatus Andrews, Dodonaea viscosa Jacq., Plectranthus ornatus Codd, Eugenia uniflora L., and Foeniculum vulgare Mill. For illness and diseases, most plants were used for problems with the digestive system (20 species), followed by the respiratory system (16 species). This community possesses a wide botanical knowledge, especially of medicinal plants, comparable to observations made in other studies with fishing communities in coastal areas of the Atlantic Forest of Brazil.

Conclusions: Ethnobotanical studies in rural-urban areas contribute to preserving local knowledge and provide information that aids in conserving the remaining ecosystems in the region.

Keywords: Ethnobotany, Plant resources, Artisanal fishers, Riparian community, Rio Grande do Sul

\section{Background}

Local knowledge is being lost by many cultures in areas of high biodiversity, especially when activities related to the use of natural resources are gradually abandoned in favor of specializing in market-related activities [1]. Research on the knowledge, use, and management of natural resources by local populations is important because it confirms the value of these cultures and contributes to the self-sufficiency of these populations.

\footnotetext{
* Correspondence: mara.ritter@ufrgs.br

${ }^{5}$ Instituto de Biociências, Departamento de Botânica e Programa de

Pós-Graduação em Botânica, Universidade Federal do Rio Grande do Sul, Av. Bento Gonçalves, 9500, Bairro Agronomia, Porto Alegre, Rio Grande do Sul 91501-970, Brazil

Full list of author information is available at the end of the article
}

Several researchers have gathered ethnobotanical knowledge from communities located in the coastal area of the Brazilian Atlantic Forest, including the knowledge and use of plants [2-8], the knowledge and use of medicinal plants [9-12], plant management [13], and fishing ecology [1], among others. However, most studies in the coastal area of the Atlantic Forest have been restricted to the southeast coast, and few have addressed fishing communities [14]. Although other studies have examined the ethnobotany of similar groups that practice artisanal fishing (which are characterized by small scale production, the use of small vessels, and work within the immediate family, other relatives or neighborhood groups in which the produce is usually sold to intermediaries without the involvement of

\section{Biomed Central}


industry in marketing and fish processing [15], culturally, these groups can be quite different $[14,16]$.

In Rio Grande do Sul State, southern Brazil, approximately 12,200 active artisanal fishers depend on fishing as their main economic activity [17]. The most extensive lagoon system in Latin America is located on the coastal plain of the state, including the Lagoa dos Patos, also called the "mar de dentro" ["the inland sea"] [18]. Depending on location, fishers living in the lagoon area do not fish in the ocean but share limited fishing territories in the lagoon. The Lagoa dos Patos, according to Kalikoski et al. [19], is an important nursery ground for many commercially important species of fish and crustaceans. During the twentieth century, the lagoon was a center of artisanal fishing in southern Brazil and contributed significantly to national fish and shellfish production.

Urbanization is increasingly frequent in developing countries [20]. In these transformations, rural areas begin to be included in the urban area, producing a new category: rural-urban areas.

In Rio Grande do Sul, many fishing communities have experienced this process $[21,22]$. Simultaneously, several studies have examined artisanal fishing in sea $[23,24]$ and inland [25-28] waters, but none have focused on the ethnobotany of these fishing communities. The present study attempts to answer the following question: is the local botanical knowledge of the artisanal fishers of the rural-urban district of Lami still active, even since the district's insertion into the metropolitan region of Porto Alegre? In this context, the objectives are as follows: a) to characterize the history of the development of socioeconomic activities in Lami, emphasizing artisanal fishery; b) to characterize the local botanical knowledge of the artisanal fishers from Lami and to determine any changes in knowledge in relation to preceding generations; and c) to characterize traditional knowledge about medicinal plants and identifying species that are more important to the fishing communities.

\section{Methods}

\section{Study area}

The study was performed in the neighborhood of Lami, which is situated in the southern part of the city of Porto Alegre $(30 \mathrm{~km}$ from the city center, with approximate coordinates $30^{\circ} 15^{\prime} \mathrm{S}$ and $51^{\circ} 05^{\prime} \mathrm{W}$ ) (Figure 1). This neighborhood borders Guaíba Lake and is rural-urban, with small truck farms interspersed between several urban centers [29]. In addition to agriculture, small tradesmen and artisanal fishing persist because an active community of artisanal fishers exist, who are of Azorean descent. This region of the city has undergone rapid urbanization with some accompanying deforestation.

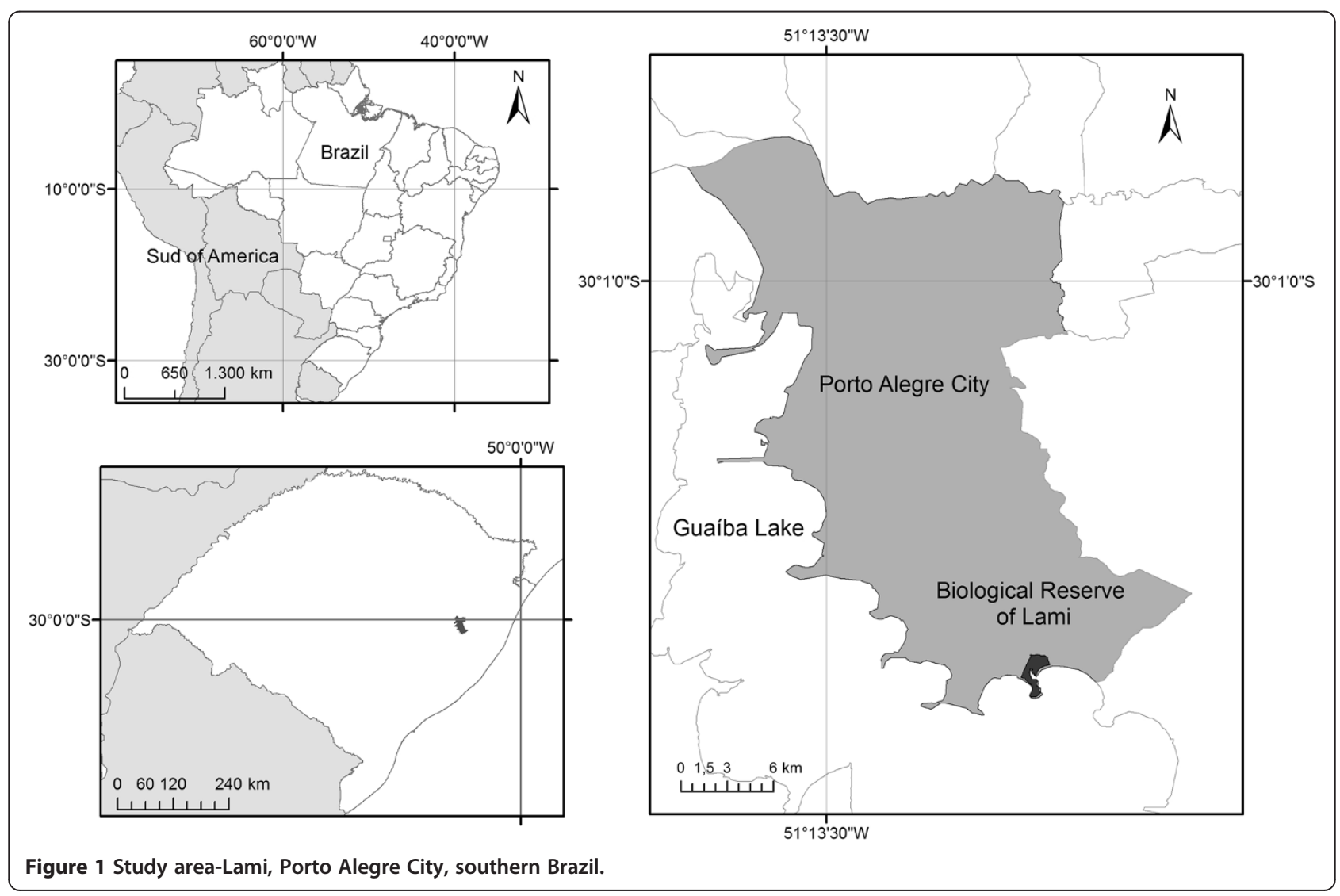


The forest remnants of Porto Alegre are located in this area, and based on the presence of these remnants, combined with the presence of the rare gymnosperm Ephedra tweediana Fisch and C.A. Mey, a municipal conservation unit was created: the Lami Biological Reserve (LBR), which protects some unique regional ecosystems and native species of fauna and flora [30]. This part of the municipality of Porto Alegre contains remnants of sandy-soil vegetation, which are characteristic of the pioneer fluvial-lacustrine formation [31].

The implementation of the LBR effectively began in 2000, when it was expanded to 179.78 ha with the preparation of the Participatory Management Plan of LBR; this plan included the participation of the fishing community and the members of the Communitarian Home-based Pharmacy of Lami (CHP) [30]. The CHP was established in 2000 as an initiative to home-produce herbal medicines and was linked to the Movement of Women Peasants of Brazil and hosted in the area of intensive use within the biological reserve. This plan provided a forum to exchange information about regional medical plants, and advice was provided by members of the academic community of the Universidade Federal do Rio Grande do Sul and nongovernmental organizations. After seven years, the Lami CHP ceased activity in 2007.

\section{Data collection}

The 13 families of the Lami fishing community were invited to participate in the study during a formal presentation of the project. All families signed a prior consent form (case number: 02000.003729/2008-29 at Conselho de Gestão do Patrimônio Genético/Ministério do Meio Ambiente/Brazil). The history of socioeconomic activity in Lami, particularly artisanal fishing, was studied by surveying literature reviews $[6,25,32,33]$ and conducting semi-structured interviews with fishers.

An ethnobotanical survey was conducted from 2007 to 2008 using participatory observation and semi-structured interviews [34,35]. Information was obtained from a script containing questions about the socioeconomic profile of the informant, the knowledge and use of plant resources in the region, methods of obtaining plant resources (cultivation, extractivism or purchase), and the details of their medicinal uses (the plant part used and the preparation method). Together with the interviews, the free-list technique was also used to assess the richness of known plants that were used by the interview subjects [35].

Fifteen fishers were interviewed (13 men and 2 women, who represented the entire community) about the knowledge and use of plants (in the categories of animal food, human food, condiments, firewood, medicinal, mystical purposes, and fishing), and the interviews were recorded and transcribed.
The plants mentioned were collected during the interviews and processed for deposition in a herbarium following standard ethnobotanical procedures [36,37]. The plants were identified in the Department of Botany, UFRGS, using specialized literature [31,38-41], and the valid plant names were confirmed using The Plant List database [42]. Collected material in good condition was deposited in the ICN herbarium of the Institute of Biosciences/UFRGS. The APG III rating system was used [43].

All the plants were classified according to habit and as native or exotic according to their biogeographical origins. We considered plants that are natural to the region (the state of Rio Grande do Sul, southern Brazil) as native species and those originating from other regions of Brazil, South America, and other continents as exotic species.

The studied species were categorized according to their uses: medicinal, human food, animal food, condiments, firewood, mystical purposes, and fishing. In the last group, we included plants that were used to build boats and temporary fishing camps, to tan cotton fishing nets, and to make buoys (or floats).

The medicinal uses mentioned by the fishers were classified according to disease categories mentioned in the ICD-10-International Statistical Classification of Diseases and Related Health Problems of the World Health Organization [44] (Table 1). In this sense, the uses mentioned by the interview subjects were grouped into 17 categories.

\section{Data analysis}

The chi-square test was used to evaluate comparisons between species richness and biogeographical origin, methods of obtaining plants, habits, and categories of use; differences were considered significant at $\mathrm{p}<0.05$ [45]. For each identified method of obtaining plants and for the habits and categories of use, the existence of differences in the proportions of native and exotic species was tested using a chi-square test. The same test was used to assess differences in the proportions of species and the methods of obtaining them within the usage categories that were identified. The analyses were conducted using BioEstat 5.0 software (Instituto Mamirauá, Brasília, Brazil) [45]. The use categories were analyzed by calculating the agreement of use according to Amorozo and Gély [46], in which the percentage of agreement of the main uses (AMU) was calculated to determine the relative importance of species that were mentioned by the community. The AMU was calculated using the formula: AMU = $(\mathrm{IAMU} / \mathrm{IAUS}) \times 100$, in which IAMU $=$ the number of interview subjects mentioning the main use and IAUS = the number of interview subjects who mentioned one or more uses for the species.

The AMU value was multiplied by a correction factor (CF) that is used to consider the citation frequency of 
Table 1 Categories of diseases mentioned by artisanal
fishers of the Lami neighborhood, Porto Alegre, Rio Grande do Sul, Brazil

\begin{tabular}{|c|c|}
\hline Category of disease & Mentioned medicinal use \\
\hline Abortifact & Abortifact \\
\hline Aphrodisiac & Aphrodisiac \\
\hline Mouth and throat & Toothache, gargling, mouth infection \\
\hline $\begin{array}{l}\text { Nutrition and } \\
\text { metabolism }\end{array}$ & $\begin{array}{l}\text { Cholesterol, diabetes, weight loss, } \\
\text { triglycerides }\end{array}$ \\
\hline Skin & Chilblains, wart removal \\
\hline Circulatory system & $\begin{array}{l}\text { Blood thinning, anemia, raised blood } \\
\text { pressure, low blood pressure, heart, to } \\
\text { unblock veins, palpitations, pressure } \\
\text { regulation }\end{array}$ \\
\hline Digestive system & $\begin{array}{l}\text { Stomach, colic, congestion, diarrhea, } \\
\text { abdominal pain, stomach ache, nausea, } \\
\text { stomach, liver, gas, laxative, malaise, } \\
\text { intestinal problems, antidiarrheal }\end{array}$ \\
\hline Genitourinary system & $\begin{array}{l}\text { Bladder, cystitis, diuretic, inflammation of } \\
\text { kidneys and bladder, urinary tract infection, } \\
\text { menopause, increasing lactation, kidney } \\
\text { stones, kidney, uterus }\end{array}$ \\
\hline Nervous system & Calmative \\
\hline $\begin{array}{l}\text { Musculoskeletal system } \\
\text { and conjunctive tissue }\end{array}$ & Hemorrhoids \\
\hline Respiratory system & $\begin{array}{l}\text { Bronchitis, catarrh, expectorant, influenza, } \\
\text { acute influenza, twinge, cold, cough }\end{array}$ \\
\hline Parasitic and infectious & $\begin{array}{l}\text { Antibiotic, cystitis, fever, infection, } \\
\text { inflammation of kidneys and bladder, } \\
\text { external infection, internal infection, urinary } \\
\text { tract infection, vermifuge }\end{array}$ \\
\hline Poisoning & $\begin{array}{l}\text { Shingles, against poison, snake bite, insect } \\
\text { stings }\end{array}$ \\
\hline Skin lesions & $\begin{array}{l}\text { Healing, against poison, external infections, } \\
\text { skin irritation, bruising (washing), snake bite, } \\
\text { insect stings }\end{array}$ \\
\hline Neoplasms & Cancer \\
\hline Hair treatment & Hair, hair loss, itching scalp \\
\hline
\end{tabular}

each species that is related to the most-often mentioned use. Thus, CF=IAUS / number of citations for the most-often mentioned use.

The corrected AMUc was determined using the following equation: $\mathrm{AMUC}=\mathrm{AMU} \times \mathrm{CF}$. Species with an AMUc of greater than $50 \%$ have high potential for medicinal use.

\section{Results}

The artisanal fishing community of Lami comprises 15 families, in which the mean age of the subjects was 50 years, ranging from 31 to 75 years. Most subjects had not completed elementary school. Two fishers were natives of Lami, and six were from nearby regions, including other neighborhoods of Porto Alegre and the nearby municipality of Viamão. The remaining fishers were from other cities in Rio Grande do Sul. Most of the subjects had lived in Lami for several years (mean $=35)$; the oldest resident had lived there for approximately 60 years and had fished for 47 years; the newest resident had fished there for four years.

The fishers mentioned 111 plant species belonging to 50 botanical families, of which Asteraceae and Lamiaceae were the most species-rich (13 and 12, respectively) (Table 2). Both native plants (50 spp.) and exotic plants (61 spp.) were mentioned, with no significant differences between the richness of species in the two categories $\left(\chi^{2}=\right.$ 1.09; $\mathrm{p}=0.34$ ).

Most species are acquired exclusively by cultivation (64 spp.), extractivism (25 spp.), or purchase (9) (Figure 2). Significant differences were observed in these proportions $\left(x^{2}=40.75 ; \mathrm{p}<0.0001\right)$, reinforcing the importance of cultivation as the main method by which the Lami fishers acquire plants. Only 13 species were obtained in more than one way (Figure 2, Table 2).

Most native plants are acquired through extractivism (32 spp.), although a nearly identical number of native plants are cultivated (26 spp.) (Figure 2). These proportions did not differ significantly $\left(x^{2}=0.21 ; p=0.76\right)$, indicating that the use of native plants in the region cannot be understood as a primarily extractivist activity because the arboreous species are obtained by extractivism and the herbaceous species are obtained by cultivation. In contrast, most of the exotic plants are cultivated (47 spp.), and only three are obtained by extractivism in nonmanaged areas (Figure 2), with significant differences in these values $\left(x^{2}=38.72 ; \mathrm{p}<0.0001\right)$. According to our interview subjects, the exotic species collected in nonmanaged areas include the trees Cinnamomum verum, Eucalyptus sp., and Punica granatum (Table 2).

Herbaceous plants were the most species-rich group (43 spp.), followed by trees (38 spp.), shrubs (24 spp.), climbers (5 spp.), and epiphytes (1 spp.) (Figure 3). The richness of herbaceous plants was not significantly different from that of trees $\left(x^{2}=0,31 ; p=0.66\right)$ but was statistically greater than that of the remaining groups $(\mathrm{p}<$ 0.05). Thus, the proportions of herbaceous and arboreal species mentioned by members of the community are similar. Most of the herbaceous and shrubby plants mentioned are exotic (26 and 14 species, respectively), but this richness is not significantly different from the number of native plants that was recorded for these two groups (17 and 10 species, respectively) (Figure 3 ) (herbaceous: $\chi^{2}=1.88 ; \mathrm{p}=0.22$; shrubby: $\chi^{2}=0.67 ; \mathrm{p}=0.54$ ). Despite this similarity, it appears that exotic herbs and shrubs are most strongly represented in the repertoire of local knowledge. In contrast, the group of arboreal species comprises predominantly native species (26 spp.), significantly more than the richness of exotic arboreal plants (12 spp.) (Figure 3$)\left(x^{2}=5.16 ; \mathrm{p}=0.03\right)$. 
Table 2 Plant species used by the Lami fishing community

Family species/popular name

Amaranthaceae

Alternanthera brasiliana (L.) Kuntze/ penicilina, ampicilina

Amaryllidaceae

Allium ampeloprasum L./alho-poró

Allium fistulosum L./cebolinha

Allium sativum L./alho

\section{Anacardiaceae}

Schinus terebinthifolia Raddi/aroeira

\section{Annonaceae}

Annona sp./fruta-do-conde

\section{Apiaceae}

Foeniculum vulgare Mill./funcho

Petroselinum crispum (Mill.) Fuss. Hoffm./salsa

\section{Aristolochiaceae}

Aristolochia triangularis Cham./

cipó-milongo ou cipó-mil-homens

\section{Asparagaceae}

Aloe arborescens Mill./babosa

Aloe maculata All./babosa-folha-gorda

Sansevieria trifasciata Prain/ espada-de-são-jorge

\section{Asteraceae}

Achillea millefolium L./canforeira

Achyrocline satureioides (Lam.) DC./marcela

Artemisia absinthium L./losna

Baccharis sp./carqueja

Chaptalia nutans (L.) Pol./cachimbinha

Gamochaeta sp./transagem

Gochnatia polymorpha (Less.) Cabrera /cambará

Gymnanthemum amygdalinum (Delile) Sch.Bip. ex Walp. /figatil

Helianthus annuus L./girassol

Hypochaeris chillensis (Kunth) Hieron./radicci, almeirão-do-mato

Lactuca sativa L./alface

Mikania laevigata Sch. Bip. ex Baker/ guaco

Tanacetum vulgare L./ catinga-de-mulata, palma-crespa, palma-de-arnica

\section{Basellaceae}

Anredera cordifolia (Ten.) Steenis/ nó-de-cachorro, palma-gorda, planta-para-anemia

\section{Bignoniaceae}

Dolichandra unguis-cati (L.) L.G.Lohmann /cipó-unha-de-gato

Handroanthus heptaphyllus (Vell.) Mattos /ipê-roxo

\section{Bromeliaceae}

Bromelia antiacantha Bertol./ bananinha-do-mato

\section{Caricaceae}

Carica papaya L./mamoeiro

\section{Habit}

Origin

Source

Use

Part of plant used

HE

CL

ME

LE

HE

$\mathrm{CL}$

$\mathrm{CO}$

EP

HE E

$\mathrm{CL}$

$\mathrm{CO}$

EP

HE $\quad \mathrm{E}$

$\mathrm{CL}$

$\mathrm{CO}$

EP

$\begin{array}{llll}\text { AR } & \mathrm{N} & \mathrm{FI} & \mathrm{BA}\end{array}$

AR $\quad E$

$\mathrm{CL}$

$\mathrm{FO}$

FR

HE $\quad$ E

$\mathrm{CL}$

$\mathrm{ME}$

LE

HE $\quad \mathrm{E}$

$\mathrm{CL}$

CO/ME

LE/RO

CL

EX

ME

EP

$\mathrm{SH} \quad \mathrm{E}$

$\mathrm{SH} \quad \mathrm{E}$

HE E

$\mathrm{CL}$

ME

LE

$\mathrm{CL}$

ME

LE

CL

MY

LE

$\mathrm{SH}$

HE

HE

HE

HE

HE

AR

$\mathrm{SH}$

$\mathrm{SH}$

HE

HE

HE

CL

CL

HE

CL N

$\mathrm{CL}$

ME

PU/EX

ME

$\mathrm{CL}$

EX

EX

$\mathrm{CL}$

$\mathrm{CL}$

PU

CL

PU

EX

CL

CL

CL

$\mathrm{CL}$

CL/EX

FO/ME

LE/ST

CL N

EX

ME

EP

AR N

PU

FI

ST/AP

HE

N

EX

FO/ME

FR

E

CL

FO

FR 
Table 2 Plant species used by the Lami fishing community (Continued)

\section{Combretaceae}

Terminalia australis Cambess./ amarilho

\section{Convolvulaceae}

Ipomoea batatas (L.) Poir./ batata-doce

\section{Costaceae}

Costus spiralis (Jack.) Roscoe/cana-do-brejo

\section{Cucurbitaceae}

Citrullus lanatus (Thunb.) Matsum. \& Nakai/melancia

Cucumis melo L./melão

Sechium edule (Jack.) Sw./chuchu

\section{Equisetaceae}

Equisetum hyemale L./cavalinha

\section{Erythroxylaceae}

Erythroxylum argentinum O. E. Schulz/cocão

\section{Euphorbiaceae}

Euphorbia prostrata Aiton/ quebra-pedra-rasteiro

Euphorbia tirucalli L./avelã

Manihot esculenta Crantz/aipim

Sebastiania schottiana (Müll. Arg.) Müll. Arg./ sarandi

Sebastiania sp./branquilho

\section{Fabaceae}

Apuleia leiocarpa (Vogel) J.F. Macbr./ grápia

Enterolobium contortisiliquum (Vell.) Morong./timbaúva

Erythrina crista-galli L./corticeira

Inga virescens Benth./angazeiro

Mimosa bimucronata (DC.) Kuntze/ maricá

Myrocarpus frondosus Allemão/ cabriúva

Parapiptadenia rigida (Benth.) Brenan/angico

\section{Geraniaceae}

Pelargonium graveolens L' Hér./ malva-crespa, malva-cheirosa

\section{Lamiaceae}

Cunila microcephala Benth./poejo

Melissa officinalis L./menta

Mentha sp.1/hortelã-branca

Mentha sp.2/hortelã

Ocimum americanum L./manjericão

Ocimum carnosum (Spreng.) Link \& Otto ex Benth. /arnica, aniz

Ocimum gratissimum L./cravo

Origanum majorana L./manjerona

Origanum vulgare L./orégano

Plectranthus barbatus Andrews/boldo

Plectranthus ornatus Codd/boldo-chileno, boldo-chinês, boldo-cheiroso

Rosmarinus officinalis $L$./alecrim

Vitex megapotamica (Spreng.) Moldenke/trauma

\begin{tabular}{|c|c|c|c|c|}
\hline$A R$ & $N$ & EX & $\mathrm{Fl}$ & $\mathrm{BA}$ \\
\hline HE & $E$ & $\mathrm{CL}$ & $\mathrm{FO}$ & $\mathrm{RO}$ \\
\hline $\mathrm{SH}$ & $E$ & $\mathrm{CL}$ & ME & $\mathrm{AP} / \mathrm{LE}$ \\
\hline $\mathrm{HE}$ & $E$ & $\mathrm{CL}$ & $\mathrm{FO}$ & $\mathrm{FR}$ \\
\hline HE & $\mathrm{E}$ & $\mathrm{CL}$ & $\mathrm{FO}$ & $F R$ \\
\hline $\mathrm{CL}$ & $E$ & $\mathrm{CL}$ & $\mathrm{FO} / \mathrm{ME}$ & LE/FR \\
\hline HE & $E$ & $\mathrm{CL}$ & ME & EP \\
\hline$A R$ & $N$ & CL/EX & FW/ME/FI & $\mathrm{BA} / \mathrm{AP}$ \\
\hline HE & $N$ & $\mathrm{CL}$ & ME & LE \\
\hline $\mathrm{SH}$ & $E$ & $\mathrm{CL}$ & ME & AP \\
\hline HE & $\mathrm{E}$ & $\mathrm{CL}$ & $\mathrm{FO}$ & $\mathrm{RO}$ \\
\hline AR & $N$ & EX & $\mathrm{FI}$ & $\mathrm{AP}$ \\
\hline AR & $N$ & EX & FW & AP \\
\hline$A R$ & $N$ & PU & $\mathrm{Fl}$ & ST/AP \\
\hline AR & $N$ & PU/EX & $\mathrm{FI}$ & ST/AP \\
\hline$A R$ & $N$ & EX & $\mathrm{Fl}$ & ST \\
\hline AR & $N$ & EX & $\mathrm{Fl}$ & BA \\
\hline $\mathrm{SH}$ & $N$ & EX & FW & AP \\
\hline AR & $N$ & PU & $\mathrm{FI}$ & ST/AP \\
\hline$A R$ & $N$ & PU & $\mathrm{Fl}$ & ST/AP \\
\hline SH & $\mathrm{E}$ & $\mathrm{CL}$ & ME & LE \\
\hline HE & $N$ & $\mathrm{CL}$ & ME & LE \\
\hline $\mathrm{HE}$ & $\mathrm{E}$ & $\mathrm{CL}$ & ME & LE \\
\hline $\mathrm{HE}$ & $E$ & $\mathrm{CL}$ & CO/ME & LE \\
\hline $\mathrm{HE}$ & $E$ & $\mathrm{CL}$ & ME & LE \\
\hline $\mathrm{HE}$ & $\mathrm{E}$ & $\mathrm{CL}$ & CO/ME & $\mathrm{LE}$ \\
\hline HE & $\mathrm{E}$ & $\mathrm{CL}$ & CO/ME & LE \\
\hline $\mathrm{HE}$ & $E$ & $\mathrm{CL}$ & FO/ME & $\mathrm{LE}$ \\
\hline $\mathrm{HE}$ & $E$ & $\mathrm{CL}$ & CO/ME & LE/AP \\
\hline HE & $E$ & $\mathrm{CL}$ & $\mathrm{CO}$ & LE \\
\hline SH & $\mathrm{E}$ & $\mathrm{CL}$ & ME & $\mathrm{LE}$ \\
\hline $\mathrm{SH}$ & $\mathrm{E}$ & $\mathrm{CL}$ & ME & $\mathrm{LE}$ \\
\hline SH & $\mathrm{E}$ & $\mathrm{CL}$ & CO/ME & LE \\
\hline $\mathrm{SH}$ & $N$ & EX & FO/ME & $B A / L E$ \\
\hline
\end{tabular}


Table 2 Plant species used by the Lami fishing community (Continued)

\section{Lauraceae}

Cinnamomum verum J.Presl (Blume)/ canela-de-casca

Laurus nobilis L./louro

AR $\quad E$

Persea americana Mill./abacate

AR

$E$

EX

FO

BA

Lythraceae

Punica granatum L./romã

Malpighiaceae

Malpighia emarginata DC./acerola

Malvaceae

Malva sylvestris $L$./malva

Sida rhombifolia L./guaxumba

\section{Melastomataceae}

Leandra australis (Cham.) Cogn./ pixirica, agulhada

\section{Meliaceae}

Cedrela fissilis Vell./cedro

HE $\quad$ E

HE N

$\mathrm{CL}$

$$
\mathrm{CO}
$$

$$
\mathrm{CL}
$$

ME

FR/SE

\section{Moraceae}

Ficus cestrifolia Schott /figueira

Maclura tinctoria (L.) D. Don ex Steud./tajuva

Morus nigra L./amoreira

(

AR

AR

$A R$
$A R$

AR

Musaceae

Musa x paradisiaca L./bananeira

\section{Myrtaceae}

Eucalyptus sp./eucalipto

Eugenia uniflora L./pitangueira

Myrciaria cuspidata O. Berg/camboim

Plinia peruviana (Poir.) Govaerts / jabuticabeira

Psidium cattleianum Afzel. ex Sabine/araçá-roxo

Psidium guajava L./goiabeira

\section{Oxalidaceae}

Averrhoa carambola L./carambola

\section{Passifloraceae}

Passiflora sp./maracujá

$\begin{array}{lllll}\text { SH } & N & \mathrm{PU} & \text { FO/ME } & \text { FR } \\ \text { HE } & N & \mathrm{CL} & \mathrm{ME} & \mathrm{LE} \\ \mathrm{HE} & \mathrm{E} & \mathrm{CL} & \mathrm{ME} & \mathrm{EP} \\ \mathrm{AR} & \mathrm{E} & \mathrm{PU} & \mathrm{Fl} & \mathrm{ST} / \mathrm{AP} \\ \mathrm{HE} & \mathrm{N} & \mathrm{CL} & \mathrm{ME} & \mathrm{LE} \\ \mathrm{HE} & \mathrm{N} & \mathrm{EX} & \mathrm{FO} / \mathrm{ME} & \mathrm{EP} \\ \mathrm{HE} & \mathrm{N} & \mathrm{CL} & \mathrm{ME} & \mathrm{EP} \\ & & & & \\ \mathrm{HE} & \mathrm{E} & \mathrm{CL} & \mathrm{FO} & \mathrm{FR}\end{array}$

\section{Phyllanthaceae}

Phyllanthus tenellus Roxb./ quebra-pedra-em-ramo

\section{Phytolacaceae}

Petiveria alliacea L./guiné

\section{Pinaceae}

Pinus sp./pinheiro

\section{Piperaceae}

Piper sp./pariparoba

\section{Plantaginaceae}

Plantago tomentosa Lam./ transagem

Plantago sp./transagem

\section{Poaceae}

(n)


Table 2 Plant species used by the Lami fishing community (Continued)

\begin{tabular}{|c|c|c|c|c|c|}
\hline Cymbopogon citratus (DC.) Stapf/ capim-cidró & HE & $E$ & $\mathrm{CL} / \mathrm{PU}$ & ME & LE \\
\hline Saccharum officinarum L./ cana-de-açúcar & $\mathrm{SH}$ & $\mathrm{E}$ & $\mathrm{CL}$ & $\mathrm{AF}$ & ST \\
\hline Zea mays L./milho & $\mathrm{SH}$ & $E$ & $\mathrm{CL}$ & $\mathrm{FO} / \mathrm{ME}$ & $\mathrm{FL}$ \\
\hline \multicolumn{6}{|l|}{ Polygonaceae } \\
\hline Persicaria hydropiperoides (Michx.) Small /erva-de-bicho & HE & $\mathrm{N}$ & EX & ME & LE/EP \\
\hline \multicolumn{6}{|l|}{ Polypodiaceae } \\
\hline Microgramma sp./cipó-cabeludo & EP & $\mathrm{N}$ & CL/EX & ME & EP \\
\hline \multicolumn{6}{|l|}{ Primulaceae } \\
\hline Myrsine guianensis (Aubl.) Kuntze/ capororoca & AR & $\mathrm{N}$ & EX & $\mathrm{FI}$ & ST/BA/AP \\
\hline \multicolumn{6}{|l|}{ Rubiaceae } \\
\hline Coffea arabica L./café & $A R$ & $E$ & $\mathrm{CL}$ & $\mathrm{FO}$ & $\mathrm{FR}$ \\
\hline \multicolumn{6}{|l|}{ Rutaceae } \\
\hline Citrus reticulata Blanco /bergamoteira & AR & $E$ & $\mathrm{CL}$ & $\mathrm{FO}$ & $\mathrm{FR}$ \\
\hline Citrus sinensis (L.) Osbeck/laranjeira & AR & $E$ & $\mathrm{CL} / \mathrm{PU}$ & $\mathrm{FO} / \mathrm{ME}$ & LE/FR \\
\hline Citrus sp./limão & $A R$ & $E$ & $\mathrm{CL} / \mathrm{PU}$ & ME & LE \\
\hline Ruta sp./arruda & $\mathrm{HE}$ & $E$ & $\mathrm{CL}$ & ME/MY & LE/EP \\
\hline \multicolumn{6}{|l|}{ Salicaceae } \\
\hline Casearia sylvestris Sw./chá-de-bugre & AR & $\mathrm{N}$ & $\mathrm{CL} / \mathrm{EX}$ & ME & $B A / L E$ \\
\hline \multicolumn{6}{|l|}{ Santalaceae } \\
\hline Jodina rhombifolia (Hook. \& Arn.) Reissek/cancorosa-de-três-pontas & $\mathrm{SH}$ & $\mathrm{N}$ & PU/EX & ME & LE \\
\hline \multicolumn{6}{|l|}{ Sapindaceae } \\
\hline \multicolumn{6}{|l|}{ Dodonaea viscosa Jacq./ } \\
\hline vassoura-vermelha & $\mathrm{SH}$ & $\mathrm{N}$ & EX & FW/ME & LE/AP \\
\hline \multicolumn{6}{|l|}{ Sapotaceae } \\
\hline Sideroxylon obtusifolium (Roem. \& Schult.) T.D. Penn./coronilho & $A R$ & $\mathrm{~N}$ & EX & ME & BA \\
\hline \multicolumn{6}{|l|}{ Solanaceae } \\
\hline Solanum lycopersicum Lam./tomate & $\mathrm{SH}$ & $E$ & $\mathrm{CL}$ & $\mathrm{FO}$ & $\mathrm{FR}$ \\
\hline Solanum sp./infalivina & $\mathrm{SH}$ & $\mathrm{N}$ & $\mathrm{CL} / \mathrm{EX}$ & ME & LE \\
\hline \multicolumn{6}{|l|}{ VERBENACEAE } \\
\hline Aloysia citrodora Palau/erva-cidreira & $\mathrm{SH}$ & $\mathrm{E}$ & $\mathrm{CL}$ & ME & LE \\
\hline Aloysia gratissima (Gillies \& Hook.) Tronc./erva-da-pontada & $\mathrm{SH}$ & $\mathrm{N}$ & $\mathrm{CL}$ & ME & LE \\
\hline
\end{tabular}

Habit: $S H$ shrub, $A R$ tree, EP epiphyte, $H E$ herbaceous, $C L$ climbing.

Origin: $E$ exotic, $\mathrm{N}$ native.

Source: $C L$ cultivation, $P U$ purchase, EX extractivism.

Use: $A F$ animal food, FO human food, $C O$ condiment, $F W$ firewood, $M E$ medicinal, $M Y$ mystical, FI fishing.

Part of plant used: $S T$ stem, $B A$ bark/HU hull, FL floral parts, $L E$ leaf, $F R$ fruit, $A P$ aerial parts, $E P$ entire plant, $R O$ root, $S E$ seed.

Most of the plants mentioned by the fishers were collected for medicinal use (69 spp.), followed by use as human food (32 spp.) and use in activities related to fishing (17 spp.) (Figure 4). All 15 interviewed fishers cited plants in the medicinal category. The richness of medicinal species mentioned was higher than that of all other categories identified in this study $(\mathrm{p}<0.05)$. This finding can be understood if we consider that the medicinal category includes a wider variety of indications of use than other categories, requiring a larger repertoire of plants for treatment.
Analysis of the relationship between the origin and the growth habit indicated that among medicinal plants, native and exotic species contribute similar proportions of species (Figure 4) $\left(\mathrm{x}^{2}=0.36 ; \mathrm{p}=0.63\right)$; this was also true for the human food category. However, species used for fishing activities and as fuel are predominantly native to the region. Plants used as condiments, for mystical purposes, and as animal food are exclusively exotic species (Figure 4).

Comparison between the usage categories and the methods of obtaining the plants showed that the Lami 


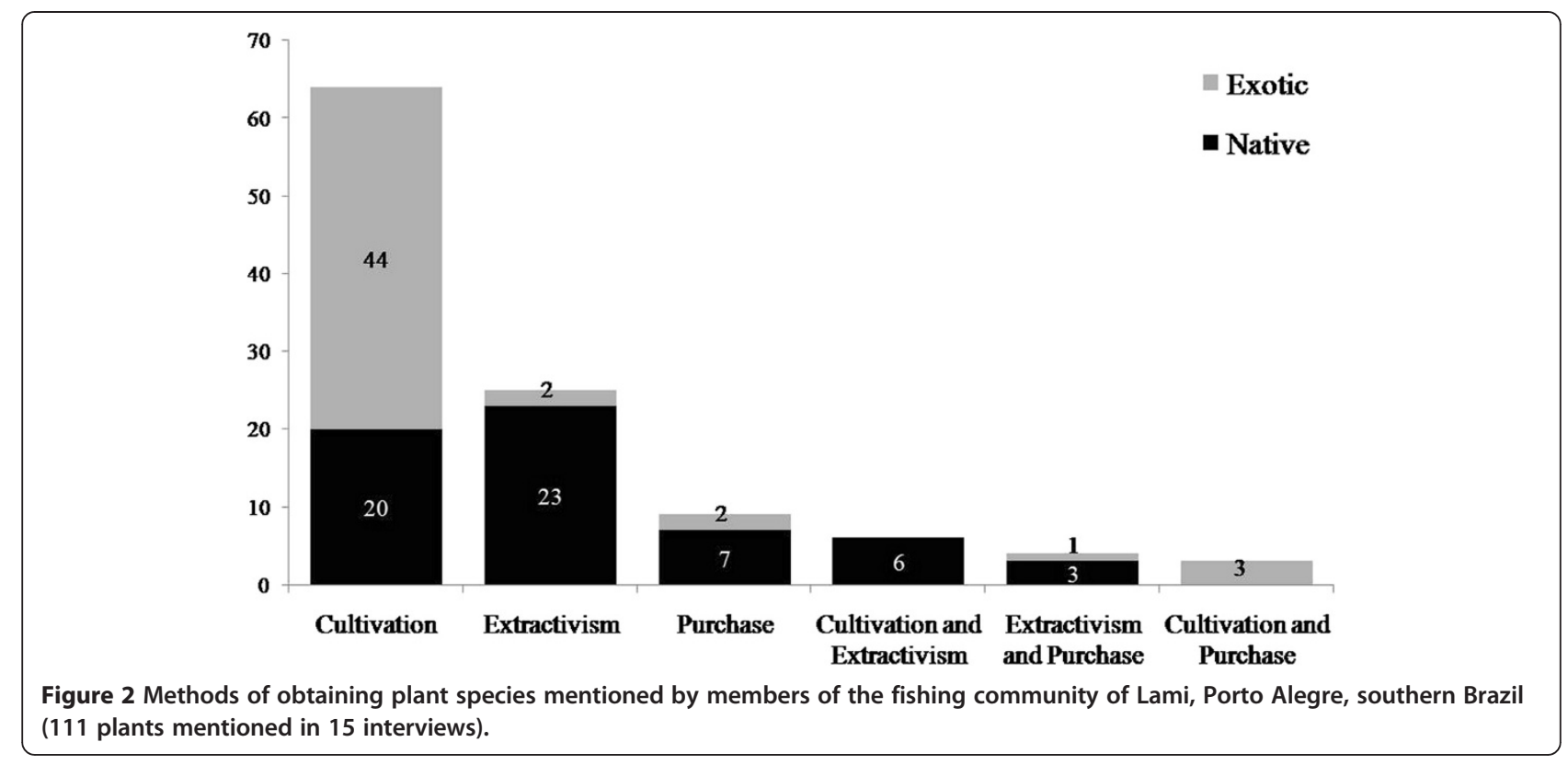

fishers obtain most of their medicinal plants through cultivation (51 spp.), followed by extractivism (21 spp.) and purchase (8 spp.) (Figure 5); these numbers were significantly different $\left(x^{2}=36.47 ; \mathrm{p}<0.0001\right)$. Extractivism was the main method used to obtain plants used for fishing and firewood (Figure 5). Few species are used for firewood, i.e., few are subject to this type of harvesting pressure. Curiously, the usage category associated with fishing included the most species acquired by purchase (Figure 5), although these were mostly native species (Figure 4).

\section{Medicinal use}

The largest numbers of medicinal plant species were used to treat ailments of the digestive system (20 spp.), followed by the respiratory system (16 spp.), genitourinary system (15 spp.), parasitic and infectious diseases (14 spp.), skin lesions (13 spp.), and diseases of the circulatory system (11 spp.) (Figure 6).

Aloe arborescens was considered the most versatile plant, being indicated for six categories of disease (Table 3), followed by Foeniculum vulgare, Cymbopogon citratus, and Aristolochia triangularis, each of which was

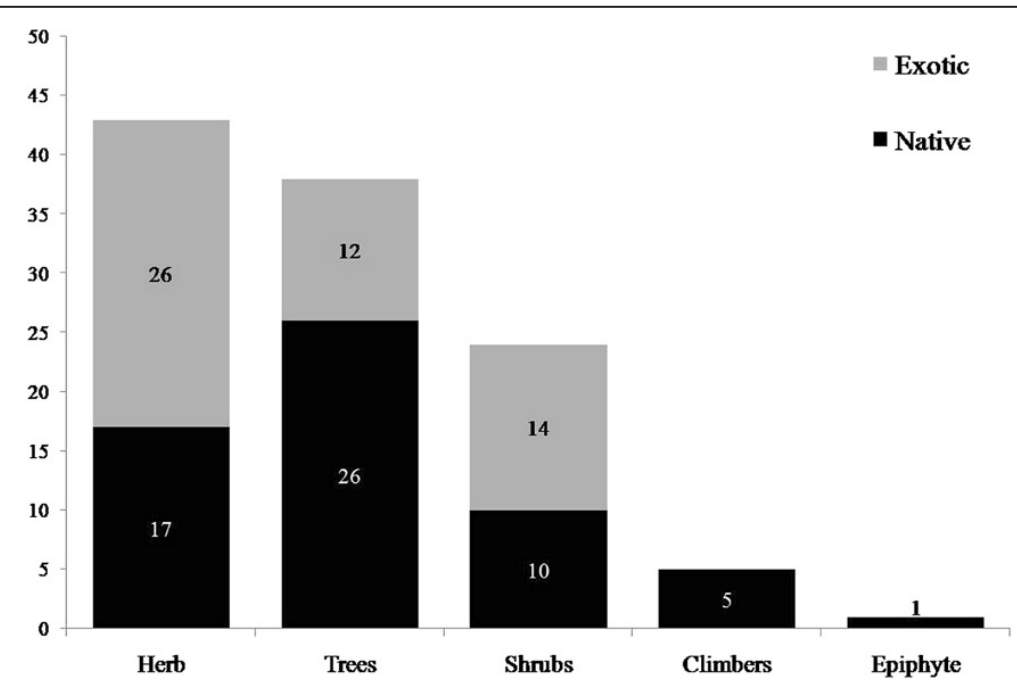

Figure 3 Richness of species grouped by habit that were recorded in the fishing community of Lami, Porto Alegre, southern Brazil (111 plants mentioned in 15 interviews). 


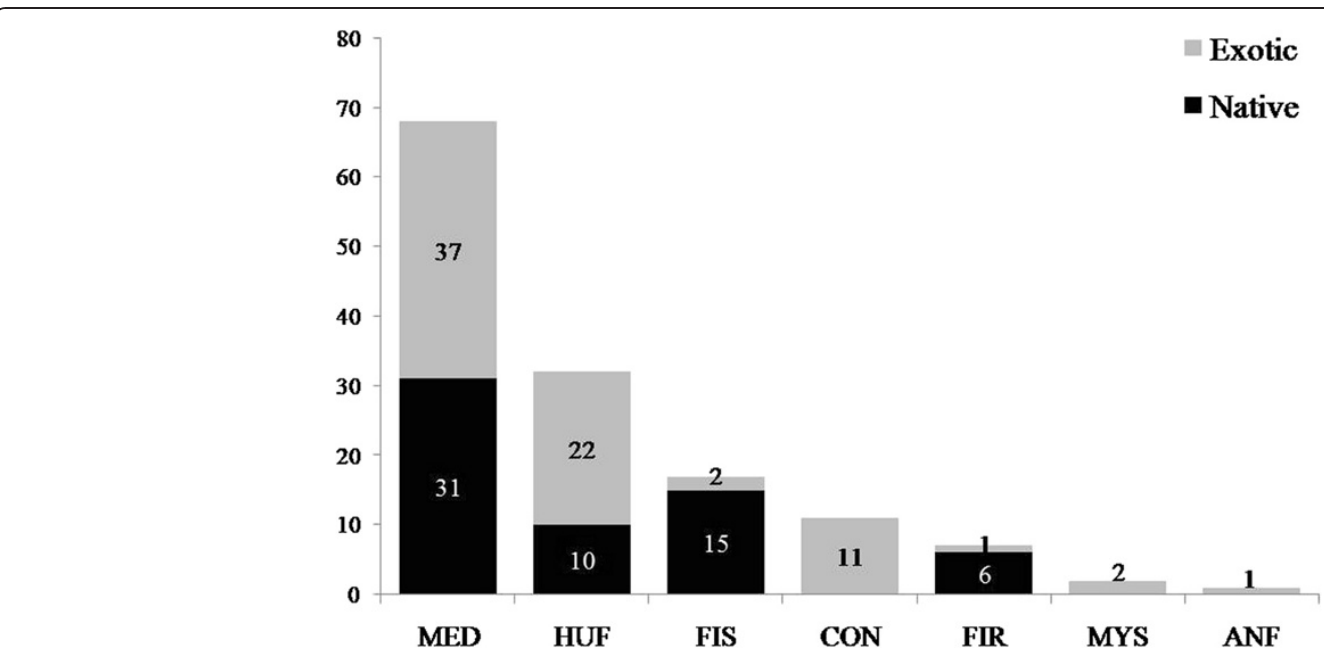

Figure 4 Usage categories and plant origin mentioned by the fishers of Lami, Porto Alegre, southern Brazil (111 plants mentioned in 15 interviews). $M E D=$ medicinal; $H U F=$ human food; FIS = fishing; $C O N=$ condiments; FIR = firewood; $M Y S=$ mystical purposes; ANF = animal food.

indicated to treat five bodily systems. However, most of the medicinal species mentioned $(65 \%)$ were indicated for only one ailment category.

Regarding the agreement on the main use (AMUc), Aloe arborescens (AMUc $=100 \%$ ) (used to treat skin lesions), as well as Plectranthus barbatus (72.73\%), Dodonaea viscosa (63.64\%), Plectranthus ornatus (63.64\%), Eugenia uniflora (54.54\%), and Foeniculum vulgare (54.54\%) (all used to treat digestive system ailments) (Table 2), exhibited high agreement. These plants may be interesting for pharmacological study regarding their possible therapeutic efficacy. Except for D. viscosa and E. uniflora, which are native plants acquired by extractivism, all of these plants are exotic cultivated species, emphasizing their importance in the local medical system.

Approximately $56 \%$ of the plants that were indicated to have medicinal properties exhibited AMUc values of less than 10\% (Table 3). These species were all cited infrequently, i.e., they were known to a few or perhaps only one person. Furthermore, most of these plants are cultivated exotic $(52 \%)$ or native species that are collected by extractivism (26\%).

\section{Use of plants for fishing}

Fishing activities can be performed daily: in the morning, when returning home for lunch, and when checking the

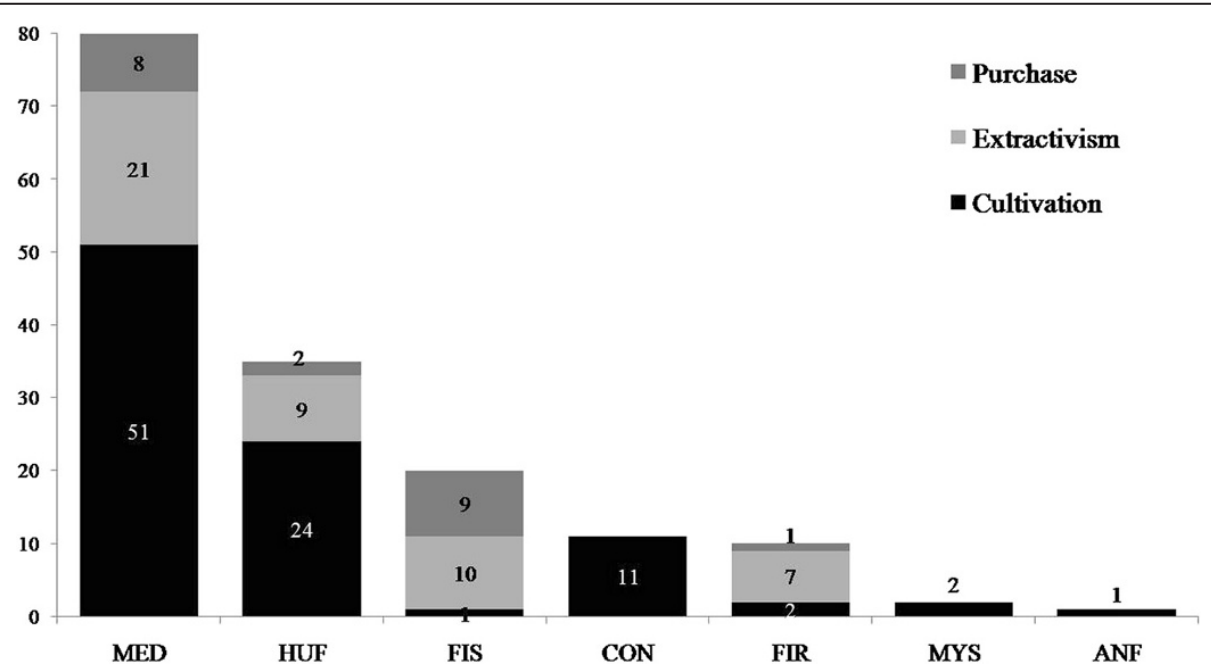

Figure 5 Usage categories and methods of obtaining plants mentioned by Lami fishers, Porto Alegre, southern Brazil (111 plants mentioned in 15 interviews). MED = medicinal; HUF = human food; FIS = fishing; CON = condiments; FIR = firewood; MYS = mystical purposes; $\mathrm{ANF}=$ animal food. 


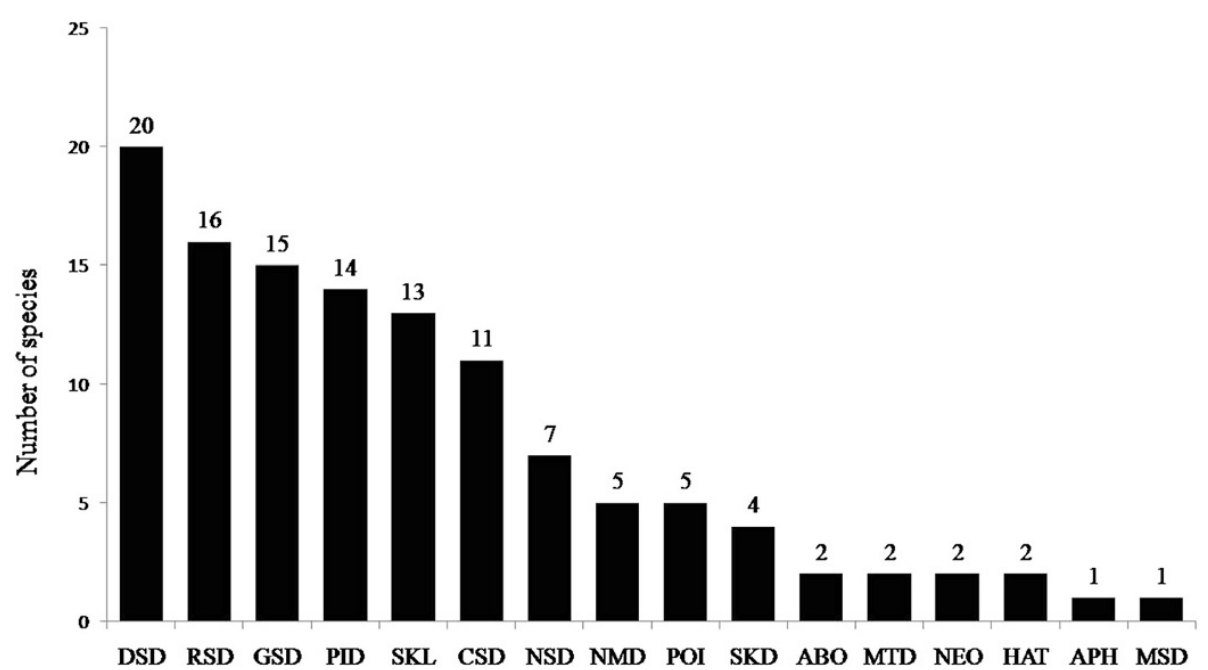

Figure 6 Distribution of medicinal species by disease category that were mentioned by the fishers of Lami, Porto Alegre, southern Brazil (69 plants mentioned in 15 interviews). $A B O=$ abortifact; $A P H=$ aphrodisiac; $M T D=$ mouth and throat diseases; NMD = nutrition and metabolism diseases; SKD = skin diseases; CSD = circulatory system diseases; DSD = digestive system diseases; GSD = genitourinary system diseases; NSD = nervous system diseases; MSD = musculoskeletal system and conjunctive-tissue diseases; RSD = respiratory system diseases; PID = parasitic and infectious diseases; $\mathrm{POI}=$ poisoning; SKL = skin lesions; NEO = neoplasms; HAT = hair treatment.

material left in the water by late afternoon or early evening. These activities can also be performed for periods of 2 to 15 days, when the fishers camp on the margins of rivers or lakes. During these periods, the fishers usually communicate with an intermediary (produce buyer), who travels every 2 or 3 days to pick up fish. Only half of the people who were interviewed use an engine; the rest fish from rowing boats [17]. The main fishing techniques are the use of sweep nets (circular fishing nets with lead weights on the extremities and a rescue rope in the center) and the use of longlines (many lines with fishing hooks) [47].

The main catches are the "bagre" (Netuma barba and N. planifrons), "cascudo" (Hypostomus spp.), "corvina" (Micropogonias furnieri), "grumatã" (Prochilodus lineatus), "jundiá" (Rhamdia spp.), "linguado" (Paralichthys spp.), "peixe-rei" (Odonthestes spp.), "piava" (Lepurinus obtusidens), "pintado" (Pimelodus maculatus), "tainha" (Mugil spp.), "traíra" (Hoplias malabaricus), and "viola" (Loricariichthys spp.) [17].

Four distinct uses of plants were identified for fishing: boat building, making fishing rods, building temporary fishing camps, and making floats for fishing nets. For boat building, the fishers mentioned the "capororoca" (Myrsine guianensis), "cambará" (Gochnatia polymorpha), "ipê-roxo" (Handroanthus heptaphyllus), "grápia" (Apuleia leiocarpa), "angico" (Parapiptadenia rigida), "pinus" (Pinus sp.), "cabriúva" (Myrocarpus frondosus), "eucalipto" (Eucalyptus sp.), "timbaúva" (Enterolobium contortisiliquum), and "cedro" (Cedrela fissilis). Some plants that were used as material for boat construction could not be identified because they are purchased by the fishers; these plants were mentioned by the names "canela-preta", "cedrinho", and "angelim". Currently, most of the wood sold in Rio Grande do Sul originates from the north and northeast of the Brazil.

For constructing fishing rods, the fishers mentioned the "sarandi" (Sebastiania schottiana), and for building temporary fishing camps, they mentioned the "capororoca" (Myrsine guianensis) and "eucalipto" (Eucalyptus sp.). According to the fishers, fishing net floats were previously made of "porongo" (Lagenaria siceraria) and "corticeira" (Erythrina crista-galli), and the weights used were stones or cattle bones. The fishing nets were previously made of cotton, but they are currently constructed from synthetic fibers.

\section{Discussion}

The similarity in the total richness of native and exotic species reveals the dynamism of the local botanical knowledge and an ability to adjust to the requirements of the local people, reflecting a pattern that has previously been documented in the literature. If the Lami fishing community live in an area of strong urban influence, this may contribute to the inclusion of species that are widely used in general society; however, if the fishers live near a biological reserve, this may allow them to have closer contact with native plants. Methods to encourage the use of exotic plants rather than native species have been discussed [48-50], but the data obtained in Lami show that the usage rate of these species is balanced. The same pattern was identified by Voeks [51] while studying the traditional pharmacopeia of an area of the Atlantic Forest in southern Bahia. 
Table 3 Agreement regarding the main medicinal uses of species

\begin{tabular}{|c|c|c|c|c|c|c|}
\hline Species & Disease category & $\begin{array}{l}\text { Number of citations } \\
\text { foruse(s) of species }\end{array}$ & $\begin{array}{l}\text { Number of citations } \\
\text { of the most-mentioned } \\
\text { use }\end{array}$ & AMU & CF & AMUC \\
\hline Aloe arborescens & $\begin{array}{l}\text { Skin lesions, hair treatment, parasitic and infectious } \\
\text { diseases, respiratory system, digestive system, } \\
\text { neoplasms }\end{array}$ & 21 & 11 & 52.4 & 1.90 & 100 \\
\hline Plectranthus barbatus & Digestive system & 8 & 8 & 100 & 0.72 & 72.72 \\
\hline Dodonaea viscosa & Digestive system & 7 & 7 & 100 & 0.63 & 63.63 \\
\hline Plectranthus ornatus & Digestive system & 7 & 7 & 100 & 0.63 & 63.63 \\
\hline Eugenia uniflora & Digestive system & 6 & 6 & 100 & 0.54 & 54.54 \\
\hline Foeniculum vulgare & $\begin{array}{l}\text { Digestive system, circulatory system, nervous } \\
\text { system, genitourinary system, nutrition and } \\
\text { metabolism }\end{array}$ & 11 & 6 & 54.5 & 1 & 54.54 \\
\hline Cunila microcephala & $\begin{array}{l}\text { Respiratory system, nervous system, parasitic and } \\
\text { infectious diseases }\end{array}$ & 7 & 5 & 71.4 & 0.63 & 45.45 \\
\hline Mikania laevigata & Respiratory system & 5 & 5 & 100 & 0.45 & 45.45 \\
\hline Achyrocline satureioides & $\begin{array}{l}\text { Digestive system, respiratory system, nervous } \\
\text { system }\end{array}$ & 8 & 5 & 62.5 & 0.72 & 45.45 \\
\hline Psidium guajava & Digestive system & 4 & 4 & 100 & 0.36 & 36.36 \\
\hline Bromelia antiacantha & Respiratory system & 4 & 4 & 100 & 0.36 & 36.36 \\
\hline Citrus sp. & Respiratory system & 4 & 4 & 100 & 0.36 & 36.36 \\
\hline Anredera cordifolia & Aphrodisiac, skin lesions, circulatory system & 5 & 4 & 80 & 0.45 & 36.36 \\
\hline $\begin{array}{l}\text { Persicaria } \\
\text { hydropiperoides }\end{array}$ & $\begin{array}{l}\text { Musculoskeletal system and conjunctive tissue, skin } \\
\text { lesions, poisoning }\end{array}$ & 5 & 4 & 80 & 0.45 & 36.36 \\
\hline Casearia sylvestris & $\begin{array}{l}\text { Circulatory system, nutrition and metabolism, } \\
\text { abortive, skin }\end{array}$ & 6 & 4 & 66.7 & 0.54 & 36.36 \\
\hline Cymbopogon citratus & $\begin{array}{l}\text { Circulatory system, digestive system, respiratory } \\
\text { system, nervous system, parasitic and infectious } \\
\text { diseases }\end{array}$ & 12 & 4 & 33.3 & 1.09 & 36.36 \\
\hline Aristolochia triangularis & $\begin{array}{l}\text { Skin lesions, poisoning, digestive system, mouth } \\
\text { and throat, circulatory system }\end{array}$ & 8 & 3 & 37.5 & 0.72 & 27.27 \\
\hline Microgramma sp. & Genitourinary system & 3 & 3 & 100 & 0.27 & 27.27 \\
\hline Citrus sinensis & Respiratory system & 3 & 3 & 100 & 0.27 & 27.27 \\
\hline Alternanthera brasiliana & Parasitic and infectious diseases & 2 & 2 & 100 & 0.18 & 18.18 \\
\hline Psidium cattleianum & Digestive system & 2 & 2 & 100 & 0.18 & 18.18 \\
\hline Persea americana & Hair treatment, skin lesions, skin & 4 & 2 & 50 & 0.36 & 18.18 \\
\hline Artemisia absinthium & Digestive system & 2 & 2 & 100 & 0.18 & 18.18 \\
\hline Sechium edule & circulatory system & 2 & 2 & 100 & 0.18 & 18.18 \\
\hline Phyllanthus tenellus & Genitourinary system & 2 & 2 & 100 & 0.18 & 18.18 \\
\hline Punica granatum & Digestive system & 2 & 2 & 100 & 0.18 & 18.18 \\
\hline Dolichandra unguis-cati & $\begin{array}{l}\text { Genitourinary system, respiratory system, digestive } \\
\text { system }\end{array}$ & 4 & 2 & 50 & 0.36 & 18.18 \\
\hline Malva sylvestris & Mouth and throat & 2 & 2 & 100 & 0.18 & 18.18 \\
\hline Euphorbia tirucalli & Neoplasms & 2 & 2 & 100 & 0.18 & 18.18 \\
\hline Aloysia citriodora & $\begin{array}{l}\text { Circulatory system, digestive system, respiratory } \\
\text { system, parasitic and infectious diseases }\end{array}$ & 5 & 2 & 40 & 0.45 & 18.18 \\
\hline Ruta sp. & Abortive, poisoning, skin lesions & 2 & 1 & 50 & 0.18 & 9.09 \\
\hline Petiveria alliacea & Poisoning, skin lesions & 1 & 1 & 100 & 0.09 & 9.09 \\
\hline Plantago sp. & Parasitic and infectious diseases & 1 & 1 & 100 & 0.09 & 9.09 \\
\hline Plantago tomentosa & $\begin{array}{l}\text { Parasitic and infectious diseases, genitourinary } \\
\text { system }\end{array}$ & 1 & 1 & 100 & 0.09 & 9.09 \\
\hline
\end{tabular}


Table 3 Agreement regarding the main medicinal uses of species (Continued)

\begin{tabular}{|c|c|c|c|c|c|c|}
\hline Aloysia gratissima & Respiratory system & 1 & 1 & 100 & 0.09 & 9.09 \\
\hline Aloe maculata & Skin lesions & 1 & 1 & 100 & 0.09 & 9.09 \\
\hline Vitex megapotamica & $\begin{array}{l}\text { Nutrition and metabolism, genitourinary system, } \\
\text { circulatory system, respiratory system }\end{array}$ & 4 & 1 & 25 & 0.36 & 9.09 \\
\hline Sida rhombifolia & Genitourinary system, circulatory system & 2 & 1 & 50 & 0.18 & 9.09 \\
\hline Ocimum carnosum & Digestive system & 1 & 1 & 100 & 0.09 & 9.09 \\
\hline Ocimum americanum & Respiratory system & 1 & 1 & 100 & 0.09 & 9.09 \\
\hline Gamochaeta sp. & Skin lesions & 1 & 1 & 100 & 0.09 & 9.09 \\
\hline Ficus cestrifolia & Skin & 1 & 1 & 100 & 0.09 & 9.09 \\
\hline Origanum majorana & Respiratory system & 1 & 1 & 100 & 0.09 & 9.09 \\
\hline Rosmarinus officinalis & Nervous system, circulatory system & 2 & 1 & 50 & 0.18 & 9.09 \\
\hline Equisetum hyemale & Circulatory system & 1 & 1 & 100 & 0.09 & 9.09 \\
\hline Piper sp. & $\begin{array}{l}\text { Genitourinary system, parasitic and infectious } \\
\text { diseases }\end{array}$ & 2 & 1 & 50 & 0.18 & 9.09 \\
\hline $\begin{array}{l}\text { Gymnanthemum } \\
\text { amygdalinum }\end{array}$ & Digestive system & 1 & 1 & 100 & 0.09 & 9.09 \\
\hline Euphorbia prostrata & Genitourinary system & 1 & 1 & 100 & 0.09 & 9.09 \\
\hline Tanacetum vulgare & Parasitic and infectious diseases, skin lesions & 2 & 1 & 50 & 0.18 & 9.09 \\
\hline Pelargonium graveolens & Parasitic and infectious diseases & 1 & 1 & 100 & 0.09 & 9.09 \\
\hline $\begin{array}{l}\text { Erythroxylum } \\
\text { argentinum }\end{array}$ & Respiratory system & 2 & 1 & 50 & 0.18 & 9.09 \\
\hline Zea mays & Genitourinary system & 1 & 1 & 100 & 0.09 & 9.09 \\
\hline Petroselinum sativum & Genitourinary system & 1 & 1 & 100 & 0.09 & 9.09 \\
\hline Chaptalia nutans & Skin lesions & 1 & 1 & 100 & 0.09 & 9.09 \\
\hline Sideroxylon obtusifolium & Nutrition and metabolism & 1 & 1 & 100 & 0.09 & 9.09 \\
\hline Costus spiralis & $\begin{array}{l}\text { Genitourinary system, parasitic and infectious } \\
\text { diseases }\end{array}$ & 1 & 1 & 100 & 0.09 & 9.09 \\
\hline Leandra australis & Digestive system & 1 & 1 & 100 & 0.09 & 9.09 \\
\hline Jodina rhombifolia & Genitourinary system, respiratory system & 2 & 1 & 50 & 0.18 & 9.09 \\
\hline Baccharis sp. & Genitourinary system, nutrition and metabolism & 1 & 1 & 100 & 0.09 & 9.09 \\
\hline Melissa officinalis & Parasitic and infectious diseases & 1 & 1 & 100 & 0.09 & 9.09 \\
\hline Mentha sp.1 & Parasitic and infectious diseases & 1 & 1 & 100 & 0.09 & 9.09 \\
\hline Morus nigra & Genitourinary system & 1 & 1 & 100 & 0.09 & 9.09 \\
\hline Solanum sp. & Digestive system, parasitic and infectious diseases & 2 & 1 & 50 & 0.18 & 9.09 \\
\hline Mentha sp.2 & Nervous system & 1 & 1 & 100 & 0.09 & 9.09 \\
\hline Ocimum gratissimum & Digestive system & 1 & 1 & 100 & 0.09 & 9.09 \\
\hline Passiflora sp. & Nervous system & 1 & 1 & 100 & 0.09 & 9.09 \\
\hline Achillea millefolium & Skin lesions, poisoning & 2 & 1 & 50 & 0.18 & 9.09 \\
\hline Helianthus annuus & Skin lesions, skin & 1 & 1 & 100 & 0.09 & 9.09 \\
\hline
\end{tabular}

In Lami, the plants obtained through cultivation are not restricted to exotic species because many native plants are obtained from fields, backyards, and community gardens. Again, this practice may be related to the presence of the Lami Biological Reserve because although the reserve contributes to the maintenance of local knowledge regarding the native flora, it also limits access to these resources, and the local community must compensate by growing culturally important native species. This response indicates the adaptive nature of traditional botanical knowledge.

The similar richness of useful herbs and trees reveals the balanced proportion of tree and herbaceous species, similar to the balance between native and exotic species. This similarity may reflect a local adaptive process that occurs because the community lives in an area where 
the use of forest resources is restricted. Thus, it is advantageous to expand the repertoire of knowledge, including species from different habits and biogeographical origins, because this expansion increases the reliability of resources: a temporarily unavailable plant can be replaced by another species. This practice is consistent with the utilitarian redundancy hypothesis, which states that the use of functionally similar species can be part of a strategy to maintain the resilience of local knowledge [48]. Thus, we found that the two most important usage categories to the community (medicinal and food) comprised similar proportions of native and exotic species.

The above discussion characterizes artisanal fishing in the studied region in that published studies have reported that useful flora tend to be dominated by exotic herbaceous species, especially in the medicinal category [49,51-54].

The use of plant species to build boats and construct fishing-net floats appears to have been an important practice in the past in various parts of Brazil. These uses have also been reported by fishers in the state of Alagoas, Northeast Brazil [55]. Traditional plants have been outmoded by new materials, resulting in the loss of this knowledge by fishers in Lami, because few fishers have reported these uses in the past. The same applies to boat building because "timbaúva" (Enterolobium contortisiliquum) and "cedro" (Cedrela fissilis) were used in Lami, and Hanazaki [14] reported that these species were also used for boat building by "caiçaras," a term used to describe traditional fishers in southern Brazil.

The predominant medicinal use of plants related to the digestive system, in many cases reflecting the population's sometimes unhealthy eating habits. Additionally, plants used to treat respiratory-system diseases were commonly mentioned, mainly because of the harsh and variable winter climate in southern Brazil. Similar data were found in other cities in Rio Grande do Sul [56,57] and other parts of Brazil [52,58-60], indicating that the residents use medicinal plants as the first line of treatment for most common diseases.

The plant described as the most versatile, Aloe arborescens, was indicated for six disease categories. $A$. arborescens is widely cultivated and used for medicinal purposes in Brazil [40].

According to floral assessments of the region's biodiversity [31,38,61-66], 594 species have been identified, of which 507 are native (85.35\%) and 87 are exotic (14.65\%). In this study, the knowledge and use of 113 species by artisanal fishers were recorded, of which 50 are native and 61 are exotic. The preference for exotic species, in principle, indicates that collection does not exert a strong pressure on native species and that use is compatible with conservation. This preference can be explained by the Azorean descent of the fishers. Rio Grande do Sul is largely influenced by European colonization, which implies the use of traditional plants of European origin, mostly for medicinal purposes. Additionally, it is perceived that most species used for medicinal purposes in the fishing community are herbaceous or bushy and more easily collected or cultivated. This pattern of use is found in many communities in Rio Grande do Sul $[56,57,67,68]$.

This paper reveals that the use pattern of plant species by the fishers who live near conservation units in ruralurban areas is compatible with the maintenance of their lifestyles and with conservation, and this use pattern should be encouraged as a conservation strategy for the co-existence of traditional populations in rural-urban areas.

\section{Conclusions}

Although the fishing community of Lami is located in a large city with easy access to modern conveniences, the fishers clearly still value and perpetuate their local botanical knowledge, mainly for medicinal use, human food, and fishing-related uses.

Our interview subjects exhibited good knowledge of the remaining vegetation in the region and of the importance of conservation. They also identified environmental degradation as a problem that affects and reduces their fishing activity.

The rescue of local knowledge and the interest of academic researchers contribute to valuing and respecting the way of life conducted by the traditional fishing community of Lami. In this community, knowledge of the past is combined with knowledge that has been recently incorporated by the community, emphasizing the cultural changes that this area of the city is experiencing.

\section{Competing interests}

The authors declare that they have no competing interests.

\section{Authors' contributions}

$M M B, G C S$, and MRR are responsible for the acquisition of study data. All authors have made substantial contributions to the conception and design of the study and to the analysis and interpretation of the data. All authors were involved in drafting and revising the manuscript and approved the final version.

\section{Acknowledgements}

The authors would like to thank the artisanal fishers of the community of Lami for their co-operation; the authors are also grateful to the CNPq for financial support (CNPq 477324/2007-0) and for the productive research scholarship to U.P. Albuquerque. The authors thank Márcia Beretta for help with the regional map. This paper is contribution number 06, supported by CAPES/PNADB through the project "Knowledge, use and conservation of plant biodiversity in the Atlantic Forest and Caatinga" (UFSC/UFRPE/UFRGS).

\section{Author details}

${ }^{1}$ Fundação Nacional do Índio, Rua Uruguai, 2648, Bairro Boqueirão, Passo Fundo, Rio Grande do Sul 99.010-112, Brazil. Universidade de Pernambuco, Campus Mata Norte, Departamento de Ciências Biológicas, Rua Prof. Amaro Maltês, 201, Sitio Novo, 55800-000, Nazaré da Mata, Pernambuco, Brazil. ${ }^{3}$ Departamento de Biologia, Área de Botânica, Universidade Federal Rural de Pernambuco, Laboratório de Etnobotânica Aplicada, Rua Dom Manuel de Medeiros s.n., Dois Irmãos, Recife, Pernambuco 52171-900, Brazil. ${ }^{4}$ Programa 
de Pós-Graduação em Desenvolvimento Rural, Universidade Federal do Rio Grande do Sul, Av. João Pessoa, 31, Centro, Porto Alegre, Rio Grande do Sul 90040-000, Brazil. ${ }^{5}$ Instituto de Biociências, Departamento de Botânica e Programa de Pós-Graduação em Botânica, Universidade Federal do Rio Grande do Sul, Av. Bento Gonçalves, 9500, Bairro Agronomia, Porto Alegre, Rio Grande do Sul 91501-970, Brazil.

Received: 30 October 2012 Accepted: 26 July 2013

Published: 30 July 2013

\section{References}

1. Hanazaki N, Begossi A: Fishing and niche dimension for food consumption of caiçaras from Ponta do Almada (Brazil). Human Ecology Review 2000, 7(2):52-62.

2. Rossato SC, Leitão-Filho HF, Begossi A: Ethnobotany of caiçaras of the Atlantic Forest coast (Brazil). Econ Bot 1999, 53(3):377-385.

3. Hanazaki N, Tamashiro JY, Leitão-Filho HF, Begossi A: Diversity of plant uses in two Caiçara communities from the Atlantic Forest Coast: Brazil. Biodivers Conserv 2000, 9(5):597-615.

4. Fonseca-Kruel VS, Peixoto AL: Etnobotânica na reserva extrativista marinha de arraial do cabo, RJ: Brasil. Acta Botanica Brasilica 2004, 18(1):177-190.

5. Miranda TM, Hanazaki N: Conhecimento e uso de recursos vegetais de restinga por comunidades das ilhas do Cardoso (SP) e de Santa Catarina (SC): Brasil. Acta Botanica Brasilica 2008, 22(1):203-215.

6. Baldauf C, Kubo RR, Silva F, Irgang BE: "Ferveu, queimou o ser da erva": conhecimentos de especialistas locais sobre plantas medicinais na comunidade do Lami, Porto Alegre, RS: Brasil. Revista Brasileira de Plantas Medicinais 2009, 11(3):282-291.

7. Borges R, Peixoto AL: Conhecimento e uso de plantas em uma comunidade caiçara do litoral sul do Estado do Rio de Janeiro: Brasil. Acta Botanica Brasilica 2009, 23(3):769-779.

8. Carneiro DB, Barboza MSL, Menezes MP: Plantas nativas úteis na Vila dos Pescadores da Reserva Extrativista Marinha Caeté-Taperaçu, Pará: Brasil. Acta Botanica Brasilica 2010, 24(4):1027-1033.

9. Di Stasi LC, Oliveira GP, Carvalhaes MA, Queiroz Junior M, Tien OS, Kakinami SH, Reis MS: Medicinal plants popularly used in the Brazilian tropical Atlantic forest. Fitoterapia 2002, 73:69-91.

10. Pinto EPP, Amorozo MCM, Furlan A: Conhecimento popular sobre plantas medicinais em comunidades rurais de mata atlântica-Itacaré, BA: Brasil. Acta Botanica Brasilica 2006, 20(4):751-762.

11. Giraldi M, Hanazaki N: Uso e conhecimento tradicional de plantas medicinais no Sertão do Ribeirão, Florianópolis/SC, Brasil. Acta Botanica Brasilica 2010, 24:395-406.

12. Meretika AHC, Peroni N, Hanazaki N: Local knowledge of medicinal plants in three artisanal fishing communities (Itapoá, Southern Brazil), according to gender, age, and urbanization. Acta Botanica Brasilica 2010, 24:386-394.

13. Adams C: As roças e o manejo da Mata Atlântica pelos caiçaras: uma revisão. Interciencia 2000, 25(3):143-150

14. Hanazaki N: Etnobotânica. In Ecologia de Pescadores da Mata Atlântica e da Amazônia: Ecologia de Pescadores da Mata Atlântica e da Amazônia. Edited by Begossi A. São Paulo: HUCITEC; 2004:37-57.

15. Diegues ACS: Populações litorâneas, movimentos sociais e ecossistemas da costa brasileira. São Paulo: USP/Cemar; 1992.

16. Diegues ACS, Arruda RA: Saberes tradicionais e biodiversidade. Brasília: Ministério do Meio Ambiente; São Paulo: USP (Biodiversidade 4); 2001.

17. Garcez D, Sánchez-Botero J: Comunidades de Pescadores Artesanais no Estado do Rio Grande do Sul: Brasil. Atlantica 2005, 27(1):17-29.

18. Toldo EE Jr: Morfodinâmica da Laguna dos Patos, Rio Grande do Sul. Pesquisas em Geociências 1991, 18(1):58-63.

19. Kalikoski DC, Rocha RD, Vasconcelos MD: Importância do conhecimento ecológico tradicional na gestão da pesca artesanal no estuário da Lagoa dos Patos, extremo sul do Brasil. Ambiente e Educação 2006, 11:87-118.

20. United Nations Human Settlements Programme: UN-Habitat Global Activities Report 2013, our presence and partnerships. http://www.unhabitat.org/pmss/ listltemDetails.aspx?publication ID $=3454$.

21. Pieve SMN, Kubo RR, Coelho-de-Souza G: A dinâmica do conhecimento ecológico local: um estudo etnoecológico sobre a resiliência dos pescadores artesanais. In VIII Reunión de Antropología del Mercosur (RAM). Buenos Aires: VIII Reunión de Antropología del Mercosur (RAM); 2009.

22. Adomilli GK, D'Ambrosio L, Carreño G, Miller FS: Povos e coletivos pesqueiros: estudos etnográficos e perspectivas socioantropológicas sobre o viver e o trabalhar. Rio Grande: Editora FURG; 2012.

23. Adomilli GK: Trabalho, meio ambiente e conflito: um estudo antropológico sobre a construção da identidade social dos pescadores do Parque Nacional da Lagoa do Peixe, RS, Msc. Thesis. Universidade Federal do Rio Grande do Sul, Programa de Pós Graduação em Antropologia Social; 2003.

24. Cotrim DS, Miguel LA: Renda da pesca artesanal: análise dos sistemas de produção na pesca em Tramandaí-RS. Redes 2009, 14(3):5-23.

25. Brutto LFG: Ecologia humana e etnoecologia em processos participativos de manejo: o caso do Parque Estadual de Itapuã, RS e os pescadores artesanais. Universidade Federal de São Carlos, Programa de Pós Graduação em Ecologia e Recursos Naturais; 2001.

26. Adomilli GK: Terra e mar, do trabalhar e do viver na pesca marítima: tempo, espaço e ambiente junto a pescadores de São José do Norte-RS, PhD Thesis. Universidade Federal do Rio Grande do Sul, Programa de Pós Graduação em Antropologia Social; 2007

27. Pasquotto VF: Pesca artesanal no Rio Grande do Sul: os pescadores de São Lourenço do Sul e suas estratégias de reprodução social, Msc. Thesis. Universidade Federal do Rio Grande do Sul, Programa de Pós-Graduação em Desenvolvimento Rural; 2005.

28. Pieve SMN: Pesca artesanal, Etnobiologia e Etnoictiologia na Colônia São Pedro (Z3), RS, Monograph. Universidade Federal de Pelotas. Curso de Ciências Biológicas; 2007.

29. Hickel HT, Albano MTF, Pavlick IMB, Bettiol D: A organização urbana: cidade rural-urbana. In Atlas ambiental de Porto Alegre. Edited by Menegat R. Porto Alegre: UFRGS; 1999:118.

30. Secretaria Municipal do Meio Ambiente: Plano de Manejo Participativo da Reserva Biológica do Lami. Porto Alegre: Prefeitura Municipal de Porto Alegre, Secretaria Municipal do Meio Ambiente; 2002.

31. Brack P, Rodrigues RS, Sobral MG, Leite SLC: Árvores e arbustos na vegetação natural de Porto Alegre. Iheringia Série Botânica 1998, 51(2):139-166

32. Grosser AJM: Percepção da qualidade ambiental: Praia do Lami, Porto Alegre/RS. In Ambiente e lugar no urbano: a grande Porto Alegre. Edited by Suertegaray DMA, Basso LA, Verdum R. Porto Alegre: Universidade/ UFRGS; 2000.

33. Rechenberg F: "Vamo falá do nosso Lami": Estudo Antropológico sobre Memória Coletiva, Cotidiano e Meio Ambiente no bairro Lami, Porto Alegre, Msc Thesis. Programa de Pós Graduação em Antropologia Social, PPGAS. Porto Alegre: Universidade/UFRGS; 2007.

34. Viertler RB: Métodos antropológicos como ferramenta para estudos em etnobiologia e etnoecologia. In Métodos de coleta e análise de dados em etnobiologia, etnoecologia e disciplinas correlatas. Edited by Amorozo MCM, Ming LC, Silva SP. Rio Claro: Divisa Gráfica Editora; 2002:11-30.

35. Albuquerque UP, Lucena RFP, Alencar NL: Métodos e técnicas para a coleta de dados etnobiológicos. In Métodos e técnicas na pesquisa etnobiológica e etnoecológica. Edited by Albuquerque UP, Lucena RFP, Cunha LVFC. Recife: NUPEEA; 2010:41-64.

36. Given DR, Harris W: Techniques and methods of Ethnobotany. London: The Commonwealth Secretariat Publications; 1994.

37. Alexiades N: Selected guidelines for ethnobotanical research: a field manual. New York: New York Botanical Garden; 1996.

38. Fuhro $D$, Vargas $D$, Larocca J: Levantamento florístico das espécies herbáceas, arbustivas e lianas da floresta de encosta da Ponta do Cego, Reserva Biológica do Lami (RBL), Porto Alegre, Rio Grande do Sul, Brasil. Pesquisas, ser. Botânica 2005, 56:239-256.

39. Sobral MG, Jarenkow JA, Brack P, Irgang BE, Larocca J, Rodrigues RS: Flora arbórea e arborescente do RS, Brasil. São Carlos: Ed. Rima/Novo Ambiente; 2006.

40. Lorenzi H, Mattos FJA: Plantas medicinais no Brasil: nativas e exóticas. Ed. Plantarum: Nova Odessa; 2008.

41. Souza VC, Lorenzi H: Botânica Sistemática. Guia ilustrado para identificação das famílias de Fanerógamas nativas e exóticas no Brasil, baseado na APG III. Ed. Plantarum: Nova Odessa; 2012.

42. The Plant List: A working list for all plants species. Version 1 (2010). 2012 Published on the internet; http://www.theplantlist.org/.

43. Stevens PF: Angiosperm Phylogeny website. 12th edition. 2012. http://www. mobot.org/MOBOT/research/APweb/. 
44. World Health Organization: International statistical classification of diseases and related health problems. http://www.who.int/classifications/apps/icd/ icd10online.

45. Ayres M, Ayres MJ, Ayres DL, Santos AAS: BioEstat 5.0: aplicações estatísticas nas áreas das ciências biológicas e médicas. Brasília: Sociedade Civil Mamirauá, CNPq; 2007.

46. Amorozo MCM, Gély A: Uso de plantas medicinais por caboclos do Baixo Amazonas, Barcarena, PA, Brasil. Boletim do Museu Paraense Emílio Goeldi, Série Botânica 1988, 4:47-131.

47. Cotrim D: Agroecologia, sustentabilidade e os pescadores artesanais: o caso de Tramandaí, Rio Grande do Sul, Msc. Thesis. Universidade Federal do Rio Grande do Sul, PGDR; 2008.

48. Albuquerque UP, Oliveira RF: Is the use-impact on native caatinga species in Brazil reduced by the high species richness of medicinal plants? J Ethnopharmacol 2007, 113:156-170.

49. Silva AJR, Andrade LHC: Etnobotânica Nordestina: estudo comparativo da relação entre comunidades e vegetação na Zona do Litoral-Mata do Estado de Pernambuco: Brasil. Acta Botanica Brasilica 2005, 19(1):45-60.

50. Case RJ, Pauli GF, Soerjato DD: Factors maintaining indigenous knowledge among ethnic communities of Manus Island. Econ Bot 2005, 59(4):356-365.

51. Voeks RA: Tropical forest healers and habitat preference. Econ Bot 1996, 50:354-373.

52. Almeida CFCBR, Amorim ELC, Albuquerque UP, Maia MBS: Medicinal plants popularly used in the Xingó region-a semi-arid location in Northeastern Brazil. J Ethnobiol Ethnomed 2006, 2(15):1-9.

53. Gazzaneo LRS, Lucena RFP, Albuquerque UP: Knowledge and use of medicinal plants by local specialists in a region of Atlantic Forest in the state of Pernambuco (Northeastern Brazil). J Ethnobiol Ethnomed 2005, 1(9):1-11.

54. Voeks RA: Disturbance pharmacopoeias: medicine and myth from the humid tropics. Ann Assoc Am Geogr 2004, 94(4):868-888.

55. Marques JGW: Pescando pescadores: etnoecologia abrangente no baixo São Francisco alagoano. São Paulo: NUPAUB-USP; 1995.

56. Kubo RR: Levantamento das plantas de uso medicinal em Coronel Bicaco, RS, Msc Thesis. Universidade Federal do Rio Grande do Sul, Programa de Pós Graduação em Botânica; 1997.

57. Garlet TMB, Irgang BE: Plantas medicinais utilizadas na medicina popular por mulheres trabalhadoras rurais de Cruz Alta, Rio Grande do Sul, Brasil. Revista Brasileira de Plantas Medicinais 2001, 4(1):9-18.

58. Hanazaki N, Leitão-Filho HF, Begossi A: Uso de recursos na Mata Atlântica: o caso da ponta do Almada (Ubatuba, Brasil). Interciencia 1996, 21(6):268-276.

59. Amorozo MCM: Uso e diversidade de plantas medicinais em Santo Antônio do Leverger, MT: Brasil. Acta Botanica Brasilica 2002, 16(2):189-203.

60. Negrelle RRB, Fornazzari KRC: Estudo etnobotânico em duas comunidades rurais (Limeira e Ribeirão Grande) de Guaratuba (Paraná, Brasil). Revista Brasileira de Plantas Medicinais 2007, 9(2):36-54.

61. Baptista LRM, Ceroni ZSV, Irgang BE, Longhi HM, Waechter JL, Miotto STS, Mariath JA, Rosito JM, Prado JF, Zanin D: Levantamento preliminar da Reserva Biológica do Lami. NIDECO Ser Urbana; 1979.

62. Foletto MC: Mapeamento de plantas exóticas na Reserva Biológica do Lami, Porto Alegre, RS.. Trabalho de conclusão de curso (graduação). Universidade Luterana do Brasil, Curso de Ciências Biológicas; 1995.

63. Silva MV, Ritter MR: Plantas medicinais e tóxicas da Reserva Biológica do Lami, Porto Alegre, Rio Grande do Sul, Brasil. Iheringia Ser Bot 2002, 57(1):61-73.

64. Brum LP: Levantamento fitossociológico do estrato arbóreo de uma mata de restinga na Reserva Biológica do Lami (RS). Trabalho de conclusão de curso (graduação). Universidade Federal do Rio Grande do Sul, Curso de Ciências Biológicas; 1995

65. Gonçalves VLC: Estudo fitossociológico do estrato arbustivo de um "vassoural" na Reserva Biológica do Lami, Porto Alegre, RS. Trabalho de conclusão de curso (graduação). Porto Alegre: Universidade/UFRGS: Curso de Ciências Biológicas; 1995.
66. Baptista MM: Etnobotânica de uma comunidade de pescadores artesanais e suas percepções sobre a Reserva Biológica do Lami, Porto Alegre, Rio Grande do Sul. Trabalho de conclusão de curso (graduação). Universidade Federal do Rio Grande do Sul, Curso de Ciências Biológicas; 2008.

67. Marodin SM, Baptista LRM: O uso de plantas com fins medicinais no município de Dom Pedro de Alcântara, Rio Grande do Sul. Revista Brasileira de Plantas Medicinais 2001, 4:57-68.

68. Vendruscolo GS, Mentz LA: Levantamento etnobotânico das plantas utilizadas como medicinais por moradores do bairro Ponta Grossa, Porto Alegre, Rio Grande do Sul, Brasil. Iheringia 2006, 61:83-103.

doi:10.1186/1746-4269-9-54

Cite this article as: Baptista et al.: Traditional botanical knowledge of artisanal fishers in southern Brazil. Journal of Ethnobiology and Ethnomedicine 2013 9:54.

\section{Submit your next manuscript to BioMed Central and take full advantage of:}

- Convenient online submission

- Thorough peer review

- No space constraints or color figure charges

- Immediate publication on acceptance

- Inclusion in PubMed, CAS, Scopus and Google Scholar

- Research which is freely available for redistribution 